\title{
Next-generation sequencing identifies novel mutations in the FBN1 gene for two Chinese families with Marfan syndrome
}

\author{
MINGJIA MA ${ }^{1 *},{\text { ZONGZHE } \text { LI }^{2 *}, \text { DAO WEN WANG }}^{2}$ and XIANG WEI ${ }^{1}$ \\ ${ }^{1}$ Division of Cardiothoracic and Vascular Surgery, Tongji Hospital; ${ }^{2}$ Division of Cardiology, Department of Internal Medicine \\ and the Genetic Diagnosis Center, Tongji Hospital, Tongji Medical College, Huazhong University of Science and Technology, \\ Wuhan, Hubei 430030, P.R. China
}

Received April 9, 2015; Accepted April 25, 2016

DOI: $10.3892 / \mathrm{mmr} .2016 .5229$

\begin{abstract}
Marfan syndrome (MFS) is an autosomal dominant heterogeneous disorder of connective tissue characterized by the early development of thoracic aneurysms/dissections, together with defects of the ocular and skeletal systems. Loss-of-function mutations in fibrillin-1 (FBN1) encoded by the gene, $F B N 1$ (MFS-1), and in the transforming growth factor $\beta$ receptor 2 (TGFBR2) gene, TGFBR2 (MFS-2), are major causes of this disorder. In the present study, a rapid and cost-effective method for genetically diagnosing MFS was described and used to identify disease-causing mutations in two unrelated pedigrees with MFS in mainland China. Using targeted semiconductor sequencing, two pathogenic mutations in four MFS patients of the two pedigrees were identified, including a novel frameshift insertion, p.G2120fsX2160, and a reported nonsense mutation, p.Arg529X (rs137854476), in the $F B N 1$ gene. In addition, a rare, probably benign Chinese-specific polymorphism in the FBNI gene was also revealed.
\end{abstract}

\section{Introduction}

Marfan syndrome (MFS, OMIM: 154700) is a fatal autosomal dominant disorder of the connective tissue with an incidence

Correspondence to: Professor Xiang Wei, Division of Cardiothoracic and Vascular Surgery, Tongji Hospital, Tongji Medical College, Huazhong University of Science and Technology, 1095 Jiefang Avenue, Wuhan, Hubei 430030, P.R. China E-mail: xiangwee_hust@163.com

Professor Dao Wen Wang, Division of Cardiology, Department of Internal Medicine and the Genetic Diagnosis Center, Tongji Hospital, Tongji Medical College, Huazhong University of Science and Technology, 1095 Jiefang Avenue, Wuhan, Hubei 430030, P.R. China

E-mail: dwwang@tjh.tjmu.edu.cn

${ }^{*}$ Contributed equally

Key words: genetic diagnosis, Marfan syndrome, next-generation sequencing, semiconductor sequencing rate of $\sim 1$ per 5,000 individuals (1). Characteristic manifestations of MFS include a series of features that involve the cardiovascular (aortic dissection, aneurysm, mitral valve prolapse, aortic root dilatation), ocular (ectopia lentis) and skeletal (special face, disproportionate slender stature, thorax deformities, scoliosis, arachnodactyly, joint hypermobility, and flat feet) systems (2). Additionally, the lungs, skin and dura may also be affected in patients with MFS. The key lethal cardiovascular symptom of patients with MFS is the development of an aneurysm or thoracic aortic dissection, particularly at an early age ( $<50$ years of age) (3). Previous studies revealed that $91 \%$ of cases of MFS were associated with fibrillin-1 (FBNI) gene mutations (MFS-1, OMIM 134797) (4), whereas a further small percentage of the patients had an underlying mutation in the transforming growth factor $\beta$ receptor 2 (TGFBR2) gene (MFS-2, OMIM 190182) (5).

Fibrillin-1, encoded by $F B N 1$, is a large, extracellular matrix glycoprotein that not only serves as an important calcium-binding microfibrillar structural molecule, but also serves as a regulator of TGF- $\beta$ signaling (6). TGFBR2 encodes a transmembrane, serine-threonine kinase domain-containing protein, TGF- $\beta$ receptor 2 . Mutations affecting the intracellular kinase domain of this protein are able to disturb TGF- $\beta$ signaling, which subsequently leads to similar features of MFS-1 (7).

The causal mutation types of the two genes include missense mutations affecting conserved residues, nonsense mutations, deletions, in-frame and splice-site mutations, and gene disruptions $(1,7)$. However, how different mutations of the genes lead to the various phenotypes of MFS has yet to be fully elucidated (8); therefore, a rapid determination of the genetic basis of MFS is vital to an improved understanding, and optimal management, of this lethal disease.

Current molecular analytical methods for MFS are single strand conformation polymorphism analysis, direct capillary sequencing and denaturing high-performance liquid chromatography. For the detection of large insertions/deletions, fluorescence in situ hybridization and multiplex ligation-dependent probe amplification are employed (4,7). Comparatively long turnaround times and/or high cost are the major disadvantages of the above-mentioned assays, with respect to their clinical use (9). Next-generation sequencing (NGS) technologies have the potential to solve these problems by rapidly dissecting 
Table I. Clinical manifestations of the two families.

\begin{tabular}{|c|c|c|c|c|}
\hline \multirow[b]{2}{*}{ General feature } & \multicolumn{3}{|c|}{ Family 1} & \multirow{2}{*}{$\frac{\text { Family } 2}{\text { Patient } 4}$} \\
\hline & Patient 1 & Patient 2 & Patient 3 & \\
\hline Age (years) & 29 & 26 & 5 & 33 \\
\hline Gender & Female & Female & Female & Male \\
\hline Height (cm) & 175 & 175 & 125 & 178 \\
\hline Weight (kg) & 63 & 67.5 & 19 & 70.5 \\
\hline \multicolumn{5}{|l|}{ Cardiovascular manifestations } \\
\hline Aortic dilatation & - & + & - & + \\
\hline Aortic dissection & - & + & - & + \\
\hline Z-score not $<2$ points & - & + & - & + \\
\hline Mitral valve prolapse & - & - & - & + \\
\hline \multicolumn{5}{|l|}{ Ocular manifestations } \\
\hline Myopia >3 diopters & - & + & - & - \\
\hline Ectopia lentis & - & - & - & - \\
\hline \multicolumn{5}{|l|}{ Systemic features } \\
\hline Thumb sign & + & + & + & + \\
\hline Wrist sign & + & + & + & + \\
\hline Pectus carinatum deformity & - & - & - & - \\
\hline Pectus excavatum or chest asymmetry & - & - & - & - \\
\hline Hindfoot deformity & - & - & - & - \\
\hline Pes planus & + & + & + & - \\
\hline Pneumothorax & - & - & - & - \\
\hline Dural ectasia & - & - & - & - \\
\hline Protrusio acetabuli & - & - & - & - \\
\hline Decreased upper body length to lower length & + & + & - & + \\
\hline Scoliosis or thoracolumbar kyphosis & - & - & - & - \\
\hline Reduced extension at elbows $<170^{\circ}$ & - & - & - & - \\
\hline Craniofacial features & + & + & + & + \\
\hline Skin striae & + & + & - & - \\
\hline Total score based on revised Ghent criteria & 7 & 8 & 5 & 6 \\
\hline
\end{tabular}

Z-score, number of standard deviations above the mean aortic root size after standardization for body surface area. Craniofacial features: enophthalmos, retrognathia, downslanting palpebral fissures.

large regions at low cost (10). The technique of semiconductor sequencing, which is rapidly gaining in popularity, is notable for having the lowest price, shortest running time, minimum start DNA amount and flexible sequencing-chip reagents (11).

The current study presents the most rapid, comprehensive, cost-efficient and reliable assay for the genetic diagnosis of MFS in routine clinical practice. By using this assay, pathogenic loss-of-function mutations in two unrelated families were identified.

\section{Materials and methods}

Ethics statement and participant recruitment. The present study was approved by the Ethics Committee of Tongji Hospital, Tongji Medical College, Huazhong University of Science and Technology (Wuhan, China), and written informed consent was obtained from each participant. The two MFS pedigrees were recruited at the Tongji Hospital between
February and August 2014. A total of 400 unrelated healthy controls were randomly recruited from healthy individuals undergoing routine health examinations in the Tongji Hospital during the corresponding period. This cohort was determined to be free of MFS by physical examination, medical history inquiry and definitive imaging examination (echocardiography or computer tomography).

\section{Case reports}

Concerning family 1: The proband (patient 2) was a 26-year-old woman who complained of an acute exacerbation of a deep, oppressive thoracodorsal pain at 31 weeks of pregnancy (Table I). After computed tomography angiography had been performed, the patient was diagnosed with a type B aortic dissection (Fig. 1). Her physical examination presented bilateral ptosis, down-slanting palpebral fissures, malar flattening and a reduced ratio of upper segment to the lower segment. The patient had mild myopia (three diopters bilateral). Her bilateral 
A

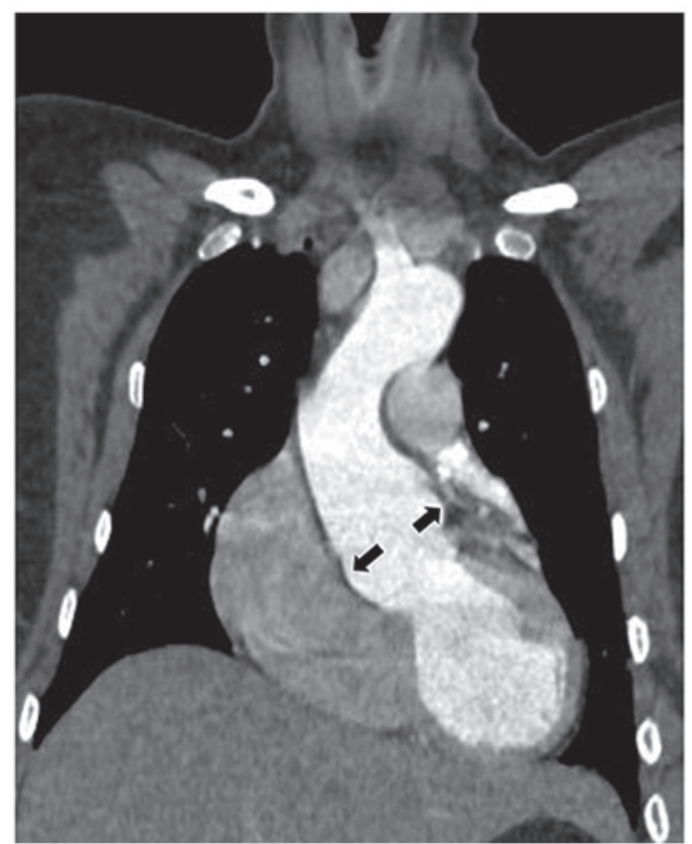

B

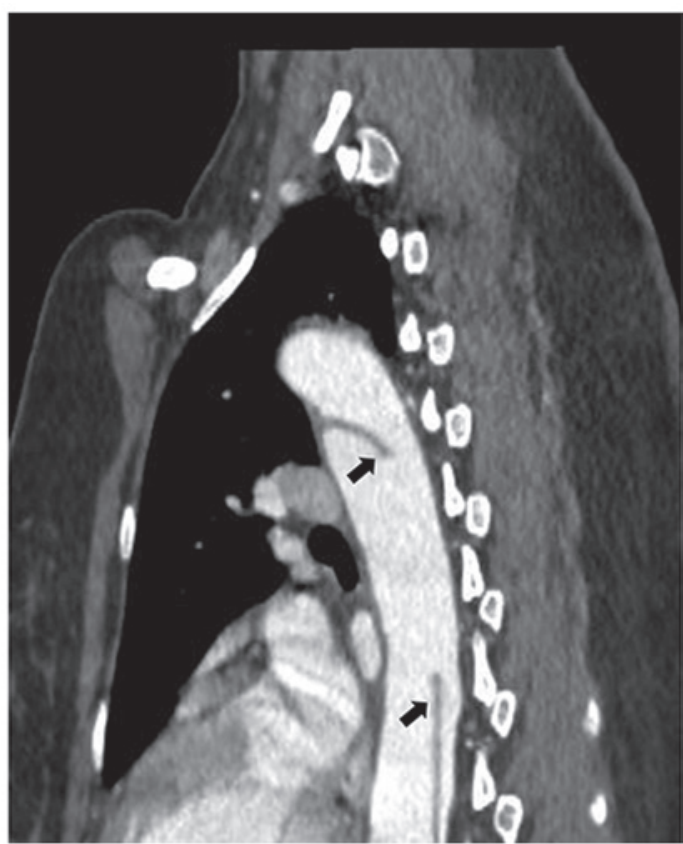

Figure 1. Chest CT scan imaging of patient 2. (A) The coronal view of chest CT scan imaging of patient 2 demonstrates dilatation at the level of the aortic sinuses (arrow labeled). (B) The sagittal view of chest CT scan of patient 2 presents the dissection of descending aorta (arrow labeled). CT, computed tomography.

'thumb signs' and 'wrist signs' were positive. The skin striae were bilateral on the shoulders, lumbar and knee regions, excluding the striae gravidarum. Echocardiography detected a dilatation of the aortic sinus to $37 \mathrm{~mm}$ without marked aortic valve regurgitation. Following a cesarean section for the delivery of an infant girl, the patient underwent replacement of the thoracic and abdominal aortas.

The elder sister (patient 1) with her 5-year-old daughter (patient 3) of the proband presented similar clinical features (Table I). The mother of the proband suffered from sudden death at the age of 45 . On the basis of the oral description and photographs, the patient (height $175 \mathrm{~cm}$, weight $67.5 \mathrm{~kg}$ ) had atypical marfanoid facial features and arachnodactyly. Other healthy relatives lacked positive phenotypical findings of MFS in family 1.

Concerning family 2: The proband (patient 4) was a 33-year-old man who presented typical marfanoid craniofacial features without ectopia lentis. The patient exhibited skeletal features such as arachnodactyly, bilateral positive wrist signs and thumb signs, and excessive spinal curvature (Table I). The patient had undergone thoracic aorta replacement due to type $\mathrm{B}$ aortic dissection 2 years previously. Echocardiography on a Vivid E9 cardiovascular ultrasound system (GE Healthcare Life Sciences, Shanghai, China) detected a dilative aortic root, with a diameter of $47 \mathrm{~cm}$. Since the dilatation of the aortic root was causing aggravation and the aortic valve developed severe regurgitation, the patient underwent a secondary operation for the aortic valve and ascending aorta replacement 1 year subsequently. His father was described to present similar clinical characteristics, and the patient suffered from sudden death at the age of 35. His mother, however, was healthy and presented with a normal phenotype.

MFS resequencing panel design and next-generation sequencing. To recruit a maximum coverage of the mutation spectrum of MFS, a specific targeted resequencing panel was designed, including two genes predisposing to MFS, FBN1 and TGFBR2 (Table II). Ion torrent adapter-ligated libraries were prepared using the Ion AmpliSeq Library kit 2.0 (Thermo Fisher Scientific, Inc., Waltham, MA, USA) following the manufacturer's protocol. The libraries of two patients with MFS were pooled together. Subsequent emulsion PCR and enrichment of the sequencing beads of the pooled libraries were performed using the Ion OneTouch 2 system (Thermo Fisher Scientific, Inc.) according to the manufacturer's protocol, as previously described (12). Finally, 500 flows (125 cycles) of sequencing were performed on the Ion 318 Chip Kit v2 using an Ion PGM Sequencing 200 Kit v2 on the Ion PGM System (Thermo Fisher Scientific, Inc.). There were 90-bp, technically uncovered regions of the two targeted genes (Fig. 2), which were carefully sequenced directly by Sanger sequencing (the primer details are shown in Table III). Prior to mutation screening, the panel was validated with four positive controls (MFS patients whose pathogenic mutations had been identified previously by Sanger sequencing).

Bioinformatics analysis. Raw data were initially processed with the Ion Torrent platform-specific software Torrent Suite $^{\mathrm{TM}}$ v. 4.2.1, to perform an alignment with the hg19 human reference genome, analyze coverage and call variants. Subsequently, all variants were annotated with an online-software Variant Effect Predictor (13). Pathogenic MFS mutations ought to be locatable in the UMD-FBN1 database (14) or in the Human Gene Mutation Database (HGMD ${ }^{\circledR}$ ) (15). To determinate likely pathogenic novel mutations, all missense substitutions were predicted and scored using the Sorting Intolerant from Tolerant (SIFT) (16) and Polymorphism Phenotyping v2 (PolyPhen-2) (17) prediction algorithms. The putative pathogenic novel mutations were further confirmed that were not in the University of California Santa Cruz 
Table II. Genes selected for Marfan syndrome-specific semiconductor sequencing.

\begin{tabular}{|c|c|c|c|c|c|c|c|}
\hline Gene & Chromosome & Ensembl & $\begin{array}{l}\text { Target } \\
\text { (bp) }\end{array}$ & $\begin{array}{l}\text { Missed }^{\mathrm{a}} \\
\text { (bp) }\end{array}$ & $\begin{array}{c}\text { Coverage }{ }^{b} \\
(\%)\end{array}$ & $\begin{array}{l}\text { Exon } \\
(\mathrm{n})\end{array}$ & $\begin{array}{l}\text { Amplicon } \\
\text { (n) }\end{array}$ \\
\hline$F B N 1$ & 15 & ENSG00000166147 & 8,588 & 28 & 99.70 & 65 & 98 \\
\hline$T G F B R 2$ & 3 & ENSG00000163513 & 1,717 & 62 & 96.50 & 8 & 16 \\
\hline
\end{tabular}

Ensembl: December 2014 (hg19). aindicates in silico missed bases by multiplex PCR; bindicates in silico coverage of target sequences by multiplex PCR. FBN1, fibrillin 1; TGFBR2, transforming growth factor $\beta$ receptor II; PCR, polymerase chain reaction.

Table III. Primers for uncovered region for Sanger sequencing

\begin{tabular}{llll}
\hline Gene & \multicolumn{1}{c}{ Position } & \multicolumn{1}{c}{ Forward primer } & \multicolumn{1}{c}{ Reverse primer } \\
\hline \multirow{2}{*}{ FBN1 } & chr15:48777666-48777693 & 5'-AGGAACCTACTGAGAGAT & 5'-ATCCCATTGAAGAAAGCA \\
& & TCAACAT-3' & CG-3' \\
TGFBR2 & chr3:30664691-30664752 & 5'-TACCAGGAAAACAGAAAA & 5'-GTGGACAAAACCCTCAAA \\
& & AAGAAGTG-3' & GAAGA-3' \\
\hline
\end{tabular}

FBN1, fibrillin 1; TGFBR2, transforming growth factor $\beta$ receptor II.

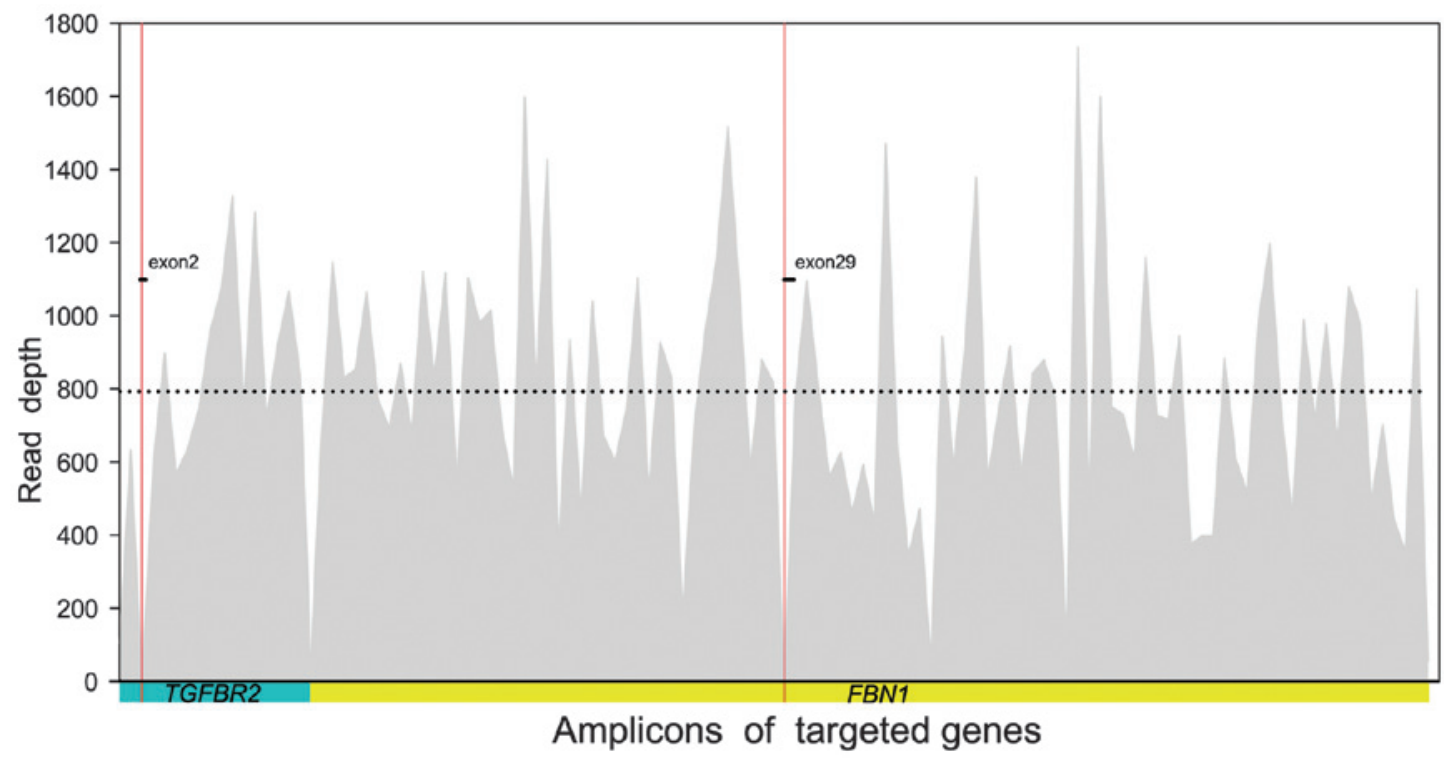

Figure 2. Semiconductor sequencing coverage overview. The diagram shows that the amplicons adequately covered the targeted genes, TGFBR2 (cyan block; NM_001024847) and FBN1 (yellow block; NM_000138) with an average read depth of 794 folds (indicated by the dotted line). The x-axis represents the distribution of amplicons; the y-axis represents the read-depth. Red areas are the technically uncovered regions, chr3:30664691-30664752 in exon 2 of the TGFBR2 gene; and chr15:4877766-48777693 in exon 29 of the FBN1 gene. FBN1, fibrillin 1; TGFBR2, transforming growth factor $\beta$ receptor II.

(UCSC) common single nucleotide polymorphism (SNP) database (18). Conservation evaluation was performed using the online software COBALT algorithm (19).

Sanger sequencing validation. All mutations detected with NGS were validated by Sanger sequencing using the BigDye Terminator v3.1 Cycle Sequencing kit (Applied Biosystems; Thermo Fisher Scientific, Inc.), followed by capillary electrophoresis on an 3500XL Genetic Analyzer (Applied Biosystems; Thermo Fisher Scientific, Inc.). The putative causal mutations were then targeted for Sanger sequencing (cascade testing) in 11 family members in family 1 , and in the mother of proband in family 2 , respectively. According to the revised Ghent nosology (1), the de novo missense mutation of FBN1 was also validated by direct Sanger sequencing in the 400 matched healthy controls to exclude pathogenic mutations in the FBN1 gene.

\section{Results}

Sequencing output and coverage. By semiconductor sequencing of selected regions of the two MFS genes in the 


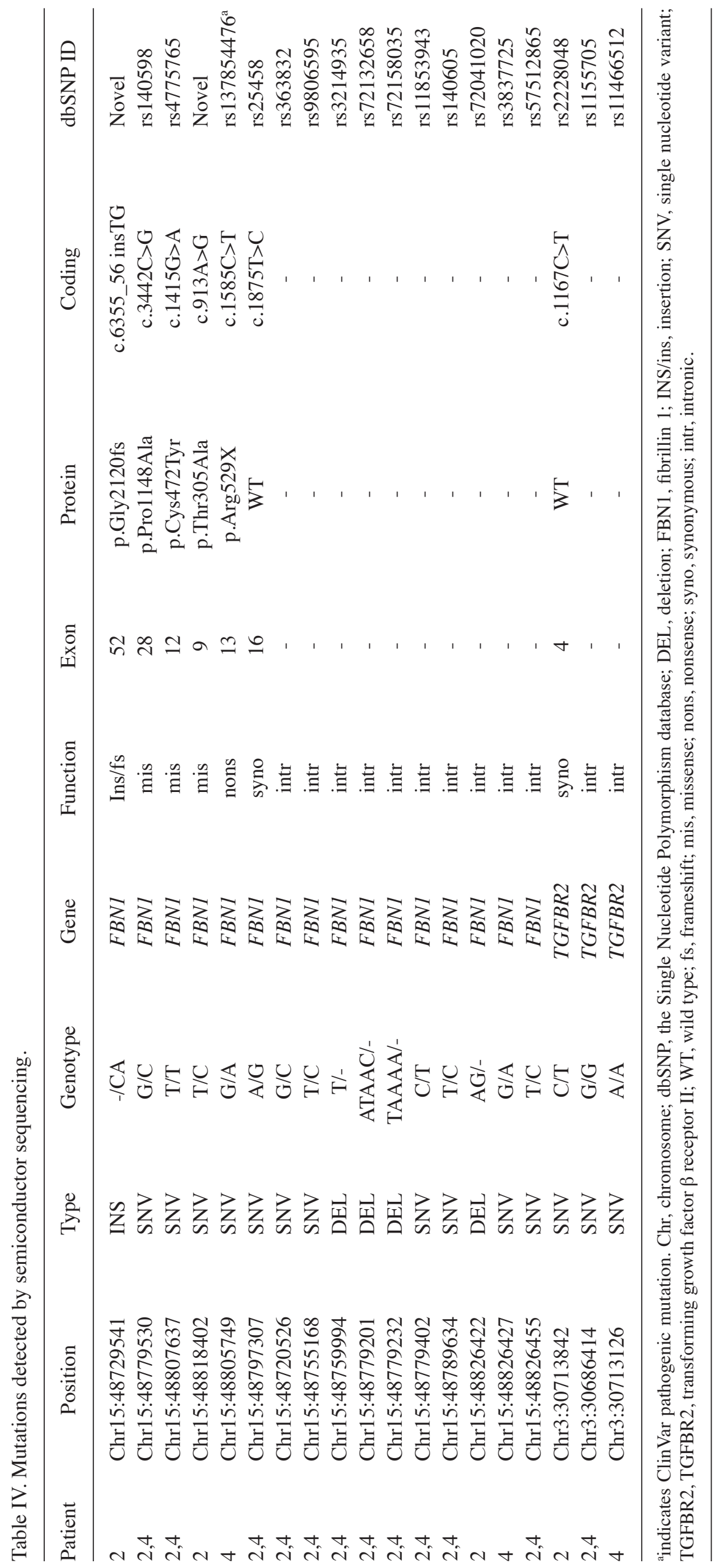



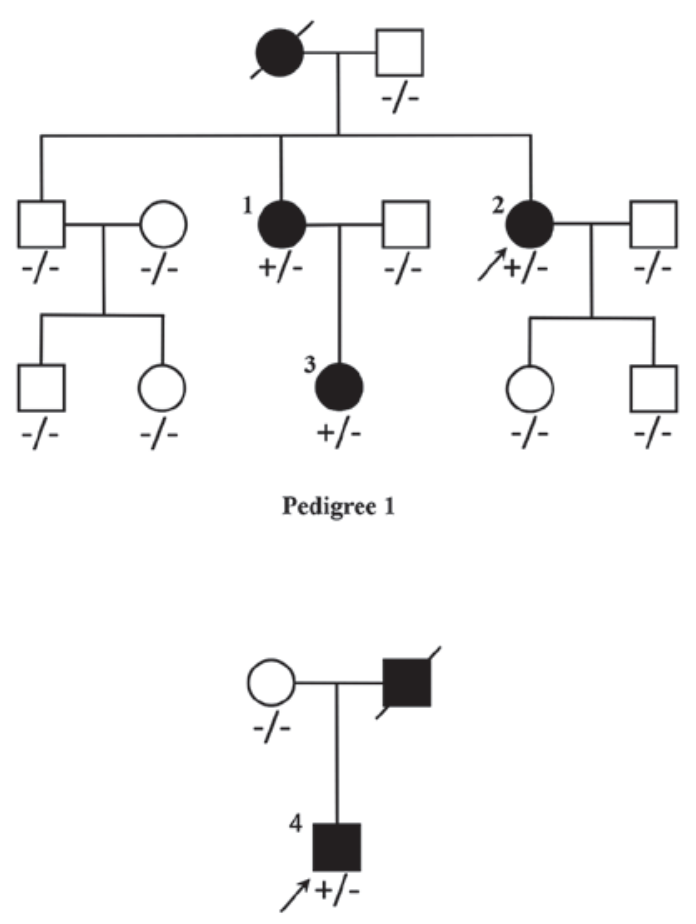

Pedigree 2

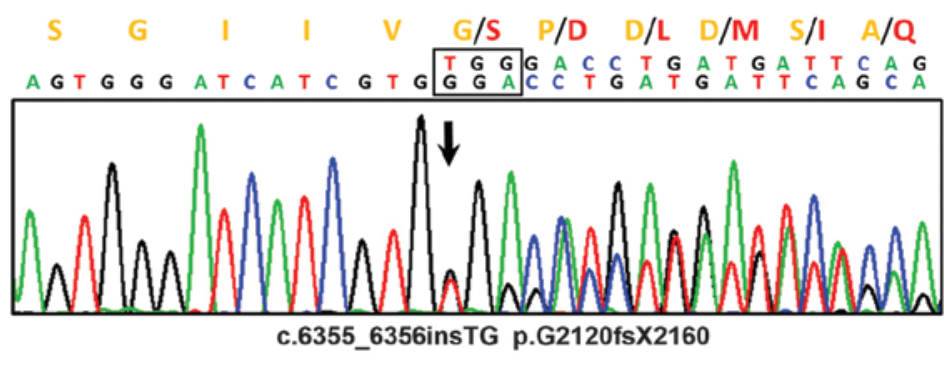

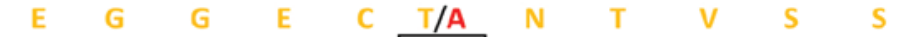
G A A G G G G G T G A A T G TAGCA A A C A C A G T C A G C A G T
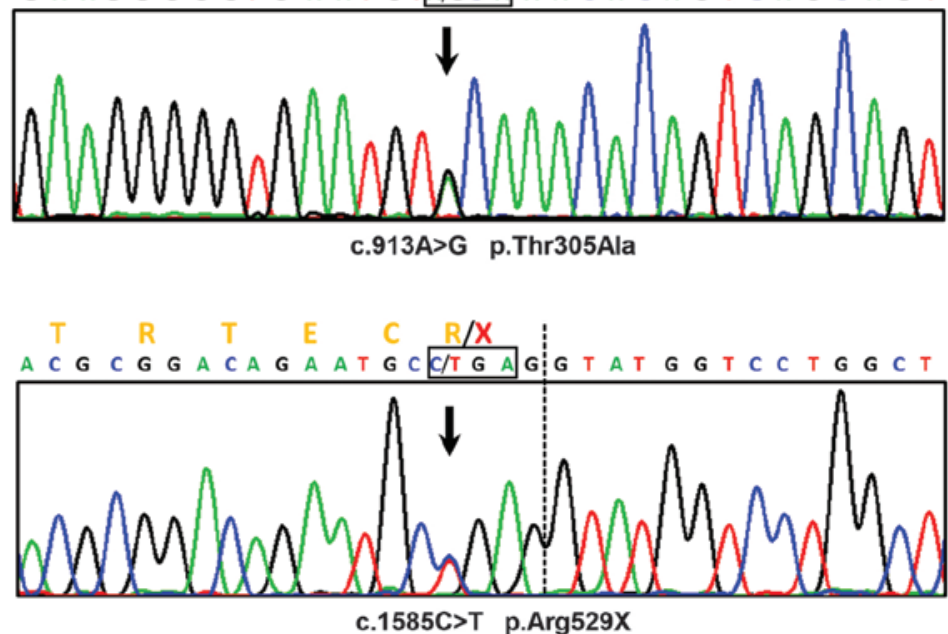

Figure 3. Pedigrees and Sanger sequence chromatograms of the detected fibrillin 1 mutations. In pedigree $1,+/-$ represents the heterozygous p.G2120fsX2160 mutation. In pedigree 2, +/- represents the heterozygous p.Arg529X mutation; -/- represents the wild type. 'Male' and 'female' are indicated by squares and circles, respectively, and the filled-in symbols represent individuals affected with Marfan syndrome. The arrow shows the proband.

probands of the two pedigrees, an average output of 589,714 mapped reads were achieved, with $89.87 \%$ on target per sample. In total, $99.11 \%$ of the amplicons were covered at least once, $97.17 \%$ of the amplicons were covered at least 20 times, and $92.14 \%$ of the amplicons were covered at least 100 times. The mean uniformity of base coverage was $91.55 \%$ in this panel. The average read-depth in the target region was 794 fold (Fig. 2).

Mutation detection and Sanger sequencing validation. In the two probands, 19 (16 in patient 2; 15 in patient 4) known or novel variants were detected by semiconductor sequencing. Following Sanger sequencing validation, all variants were determined. Of these 19 variants, 12 (63.16\%) were annotated as non-coding and $2(10.53 \%)$ were annotated as synonymous, whereas 5 (26.32\%) were non-synonymous, including three missense mutations, one nonsense mutation and one frameshift insertion, resulting in the replacements of amino acids (Table IV).

Pathogenic mutations identification. A reported MFS pathogenic nonsense mutation, c.1585C >T, p.Arg529X (rs137854476), was identified in FBN1 in patient 4 by filtering the HGMD and ClinVar databases $(15,20)$. Two novel FBN1 mutations were identified in patient 2 , including a frameshift insertion,c.6355_6356insTG(p.G2120fsX2160), and a missense mutation, c.913A $>\mathrm{G}$ (p.Thr305Ala). Neither of the mutations existed in known databases (UMD-FBN1, HGMD, ClinVar,
UCSC common SNP, dbSNP and the 1000-genome-project) or in published articles (Fig. 3).

Via cascade testing, the insertion c.6355_6356insTG was detected in patients 1 and 3 , and the remaining nine asymptomatic family members, as well as 400 healthy matched controls, consistently with the phenotype, exhibited no mutation at the identical position. The missense mutation, c.913A>G, was detected in the healthy brother of the proband (patient 2) and in two of the 400 healthy controls. Arg529X was not detected in the asymptomatic mother of patient 4 in family 2 .

Bioinformatics analysis. The de novo missense mutation c.913A $>\mathrm{G}$ underwent in silico functional analysis and a conservation test. The score of online predictive tools revealed no tendency of protein damaging (SIFT $=0.45$ and Polyphen-2=0) $(21,22)$. In the conservation test, the affected residue in patients and 13 other species was revealed to be evolutionarily non-conserved, which implied that the alternation of amino acids in this position is less likely to damage protein function. Therefore, this missense mutation was identified as being a rare, probably benign, Chinese-specific polymorphism.

\section{Discussion}

Currently, the diagnosis of MFS remains predominantly based on clinical manifestation (1). The genetic dissection of this lethal disease helps to establish the diagnosis of familial 
MFS and to predict neonatal patients and young children, even when the clinical manifestations are not yet evident (23). In the present study, a rapid and convenient measure for routine MFS genetic diagnosis has been described. With this panel and only $20 \mathrm{ng}$ input genomic DNA, physicians are able to go from blood samples to variants in a single day. Compared with the Sanger method, which takes 2 months to screen the whole FBN1 gene for 10 samples, the turnaround time of NGS is sharply reduced. The split cost of the NGS reagents was one-tenth that of the reagents required for the Sanger procedure. In addition, this MFS special panel, covering all the FBN1 and TGFBR2 coding exons and their flanking regions, simplified the data analysis procedure and was more practical for clinical use. It is the most rapid and convenient method among similar panels reported previously $(10,11,24)$. Using this assay, genotypes of two unrelated MFS pedigrees were screened. A novel pathogenic frameshift mutation, c.6355_6356insTG (p.G2120fsX2160), a reported pathogenic nonsense mutation, c.1585C >T (p.Arg529X, rs137854476), and a rare, probably benign, Chinese-specific polymorphism, c.913A $>$ G (p.Thr305Ala) in the FBN1 gene, were demonstrated.

In family 1 , three patients with MFS (patients 1,2 and 3) were demonstrated to harbor a novel FBN1 heterozygous insertion, c.6355_6356insTG, which resulted in a frameshift mutation and truncated the original 2,871 amino-acid full-length protein to a 2,160-residue protein. This mutation is in exon 52, located between TGF-like domain 8 and the epidermal growth factor (EGF)-like domain 36, according to UniProtKB (25). Since a string of amino acids was replaced and the EGF-like domains 37-47 of FBN1 were lost, our hypothesis was that there could be a severe functional defect in the mutated translation product. According to the revised Ghent criteria of causal FBN1 mutation, this insertion was able to be identified as a novel pathogenic mutation. In addition, a novel missense mutation, c.913A $>$ G (p.Thr305Ala) in FBN1, was also detected in the asymptomatic brother of the proband (patient 2) and two of the 400 negative controls. The pathogenic effect was also excluded by in silico functional analysis (SIFT=0.45, Polyphen-2=0) and a conservation test. On the basis of this evidence, it is reasonable to surmise that the novel missense mutation c. $913 \mathrm{~A}>\mathrm{G}$ is a benign SNP in the Chinese population.

In family 2 , the proband (patient 4) was demonstrated to harbor a FBN1 heterozygous nonsense mutation, c. $1585 \mathrm{C}>\mathrm{T}$, which caused translation of the protein to stop at the 529th amino acid residue. This mutation, p.Arg529X located in exon 13 , is a reported MFS pathogenic mutation, also known as rs137854476. The affected proband (patient 4) in the present study presented phenotypes which were similar to those of previous cases (26).

All patients (patients 1,2,3 and 4) in the present study fulfilled the MFS diagnostic criteria, in accordance with the genetic diagnosis results. However, since all the patients with MFS in this study were young, particularly patient 3, the typical marfanoid symptoms may not have been fully manifested. These should be monitored, since the phenotypes are variable and time-dependent.

To date, the technically uncovered regions and sequencing bias are inevitable (9). The results of NGS need to be validated using Sanger method. This panel recruited only MFS-associated genes, which limited the usefulness with respect to other inherited aortic disease diagnoses. Globally, NGS methods have achieved a comparable, or even more optimal, sequencing quality compared with standard assays.

In conclusion, the present study has provided what is, at present, the most rapid, cost-effective and reliable semiconductor MFS-specific resequencing assay for clinical routine use. A novel pathogenic insertion and a rare, probably benign Chinese-specific polymorphism in $F B N 1$ were revealed. This study enriches the mutation spectrum of $F B N 1$, and will facilitate the molecular diagnosis of MFS.

\section{Acknowledgements}

This study was funded by the National Natural Science Foundation of China (no. 81370201). We would like to thank the patients for consenting to participate in this study and publication.

\section{References}

1. Loeys BL, Dietz HC, Braverman AC, Callewaert BL, De Backer J, Devereux RB, Hilhorst-Hofstee Y, Jondeau G, Faivre L, Milewicz DM, et al: The revised Ghent nosology for the Marfan syndrome. J Med Genet 47: 476-485, 2010.

2. Drolsum L, Rand-Hendriksen S, Paus B, Geiran OR and Semb SO: Ocular findings in 87 adults with Ghent-1 verified Marfan syndrome. Acta Ophthalmol 93: 46-53, 2015.

3. Cañadas V, Vilacosta I, Bruna I and Fuster V: Marfan syndrome. Part 1: Pathophysiology and diagnosis. Nat Rev Cardiol 7: 256-265, 2010.

4. Loeys B, De Backer J, Van Acker P, Wettinck K, Pals G, Nuytinck L, Coucke $\mathrm{P}$ and De Paepe A: Comprehensive molecular screening of the FBN1 gene favors locus homogeneity of classical Marfan syndrome. Hum Mutat 24: 140-146, 2004.

5. Waldmuller S, Muller M, Warnecke H, Rees W, Schöls W, Walterbusch G, Ennker J and Scheffold T: Genetic testing in patients with aortic aneurysms/dissections: A novel genotype/phenotype correlation? Eur J Cardiothorac Surg 31: 970-975, 2007.

6. Robinson PN, Arteaga-Solis E, Baldock C, Collod-Béroud G, Booms P, De Paepe A, Dietz HC, Guo G, Handford PA, Judge DP, et al: The molecular genetics of Marfan syndrome and related disorders. J Med Genet 43: 769-787, 2006.

7. Mizuguchi T, Collod-Beroud G, Akiyama T, Abifadel M, Harada N, Morisaki T, Allard D, Varret M, Claustres M, Morisaki H, et al: Heterozygous TGFBR2 mutations in Marfan syndrome. Nat Genet 36: 855-860, 2004.

8. Faivre L, Collod-Beroud G, Loeys BL, Child A, Binquet C, Gautier E, Callewaert B, Arbustini E, Mayer K, Arslan-Kirchner M, et al: Effect of mutation type and location on clinical outcome in 1,013 probands with Marfan syndrome or related phenotypes and FBN1 mutations: An international study. Am J Hum Genet 81: 454-466, 2007.

9. Churko JM, Mantalas GL, Snyder MP and Wu JC: Overview of high throughput sequencing technologies to elucidate molecular pathways in cardiovascular diseases. Circ Res 112: 1613-1623, 2013.

10. Baetens M, Van Laer L, De Leeneer K, Hellemans J, De Schrijver J, Van De Voorde H, Renard M, Dietz H, Lacro RV, Menten B, et al: Applying massive parallel sequencing to molecular diagnosis of Marfan and Loeys-Dietz syndromes. Hum Mutat 32: 1053-1062, 2011.

11. Xiao Y, Liu Y, Yang K, Yang Y, Zhou X, Lu C, Xiao J, Liu F and Zhang $X$ : Next generation sequencing as a rapid molecular diagnosis for Marfan syndrome in a Chinese family with mutations in the fibrillin-1 gene. Clin Chim Acta 439: 58-60, 2015.

12. Li Z, Huang J, Zhao J, Chen C, Wang H, Ding H, Wang DW and Wang DW: Rapid molecular genetic diagnosis of hypertrophic cardiomyopathy by semiconductor sequencing. J Transl Med 12: 173,2014 
13. Ensembl Variant Effect Predictor: Available online: http://asia.ensembl.org/info/docs/tools/vep/index.html (accessed on 15 March 2015).

14. UMD-FBN1 database: Available online: http://www.umd. be/FBN1/ (accessed on 15 March 2015).

15. The Human Gene Mutation Database: Available online: http://www.hgmd.cf.ac.uk (accessed on 15 March 2015).

16. SIFT: Available online: http://sift.jcvi.org/ (accessed on 15 March 2015).

17. PolyPhen-2. Available online: http://genetics.bwh.harvard edu/pph2/ (accessed on 15 March 2015).

18. UCSC common SNP database. Available online: https://genome. ucsc.edu/cgi-bin/hgVai (accessed on 15 March 2015).

19. COBALT Cobalt Constraint-based Multiple Protein Alignment Tool: Available online: http://www.ncbi.nlm.nih. gov/tools/cobalt/cobalt.cgi (accessed on 15 March 2015).

20. ClinVar database. Available online: http://www.ncbi.nlm.nih. gov/clinvar/variation/16452/ (accessed on 15 March 2015).

21. Sequence SIFT: Available online: http://sift.jcvi. org/sift-bin/catfile.csh?/opt/www/sift/tmp/2996.siftresults. predictions+Predictions+PRE (accessed on 15 June 2015).
22. PolyPhen-2 report for P35555 T305A. Available online: http://genetics.bwh.harvard.edu/ggi/pph2/a914c296bdbd9ceble8afb68bef4854ebc5f1c2b/2976072.html (accessed on 15 June 2015).

23. Potter KJ, Creighton S, Armstrong L, Eydoux P, Duncan W, Penny DJ, Fan Y and Gibson WT: The c.7409G>A (p.Cys2470Tyr) Variant of FBN1: Phenotypic Variability across Three Generations. Mol Syndromol 4: 125-135, 2013.

24. Proost D, Vandeweyer G, Meester JAN, Salemink S, Kempers M, Ingram C, Peeters N, Saenen J, Vrints C, Lacro RV, et al: Performant mutation identification using targeted next generation sequencing of fourteen thoracic aortic aneurysm genes. Hum Mutat 36, 808-814, 2015.

25. UniProtKB: Available online: http://www.uniprot. org/uniprot/P35555 (accessed on 15 March 2015).

26. Rand-Hendriksen S, Tjeldhorn L, Lundby R, Semb SO, Offstad J, Andersen K, Geiran O and Paus B: Search for correlations between FBN1 genotype and complete Ghent phenotype in 44 unrelated Norwegian patients with Marfan syndrome. Am J Med Genet A 143A: 1968-1977, 2007. 